\title{
Endosymbiont diversity in natural populations of Tetranychus mites is rapidly lost under laboratory conditions
}

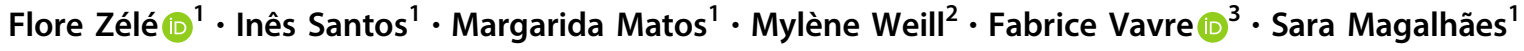

Received: 6 September 2019 / Revised: 24 January 2020 / Accepted: 24 January 2020 / Published online: 11 February 2020

(C) The Author(s), under exclusive licence to The Genetics Society 2020

\begin{abstract}
Although the diversity of bacterial endosymbionts in arthropods is well documented, whether and how such diversity is maintained remains an open question. We investigated the temporal changes occurring in the prevalence and composition of endosymbionts after transferring natural populations of Tetranychus spider mites from the field to the laboratory. These populations, belonging to three different Tetranychus species (T. urticae, T. ludeni and T. evansi) carried variable infection frequencies of Wolbachia, Cardinium, and Rickettsia. We report a rapid change of the infection status of these populations after only 6 months of laboratory rearing, with an apparent loss of Rickettsia and Cardinium, while Wolbachia apparently either reached fixation or was lost. We show that Wolbachia had variable effects on host longevity and fecundity, and induced variable levels of cytoplasmic incompatibility (CI) in each fully infected population, despite no sequence divergence in the markers used and full CI rescue between all populations. This suggests that such effects are largely dependent upon the host genotype. Subsequently, we used these data to parameterize a theoretical model for the invasion of CI-inducing symbionts in haplodiploids, which shows that symbiont effects are sufficient to explain their dynamics in the laboratory. This further suggests that symbiont diversity and prevalence in the field are likely maintained by environmental heterogeneity, which is reduced in the laboratory. Overall, this study highlights the lability of endosymbiont infections and draws attention to the limitations of laboratory studies to understand host-symbiont interactions in natural populations.
\end{abstract}

\section{Introduction}

Vertically transmitted bacterial symbionts are extremely widespread in arthropods (Gibson and Hunter 2010). While some symbiont-arthropod associations are essential for host

These authors contributed equally: Fabrice Vavre, Sara Magalhães

Supplementary information The online version of this article (https:// doi.org/10.1038/s41437-020-0297-9) contains supplementary material, which is available to authorized users.

\section{Flore Zélé}

fezele@fc.ul.pt

1 Centre for Ecology, Evolution and Environmental Changes (cE3c), Faculdade de Ciências, Universidade de Lisboa, Edificio C2, Piso-3 Campo Grande, 1749016 Lisbon, Portugal

2 Institut des Sciences de l'Evolution (CNRS-Université de Montpellier-IRD-EPHE), 34095, CEDEX 5 Montpellier, France

3 CNRS, Laboratoire de Biométrie et Biologie Evolutive UMR 5558, Université Lyon 1, Université de Lyon, F-69622 Villeurbanne, France survival and can persist for millions of years, others are facultative and are erratically distributed (reviewed in Moran et al. 2008). The maintenance of infection polymorphism of diverse facultative endosymbionts in host populations is thought to hinge mainly upon balancing selection between the costs and benefits of infection (Oliver et al. 2014). Such costs and benefits usually translate into changes in fecundity and longevity in the host. Moreover, some intracellular maternally-inherited symbionts (e.g. Wolbachia, Rickettsia, Cardinium, Arsenophonus and Spiroplasma; Duron et al. 2008; Weinert et al. 2015) are able to manipulate the reproduction of their hosts to enhance their own transmission (Engelstadter and Hurst 2009), which has important consequences for their infection dynamics. Phenotypes of reproductive manipulation include feminization, induction of thelytokous parthenogenesis, male-killing, and (the most common and best studied) cytoplasmic incompatibility (CI; Engelstadter and Hurst 2009).

In diploid species, CI leads to the embryonic mortality of part or all of the offspring resulting from crosses between infected males and uninfected females (or females infected by an incompatible strain). In contrast, crosses between infected 
females and both uninfected and infected males are fully viable, hence these females have a reproductive advantage relative to uninfected ones. This phenomenon thus allows the rapid spread of CI-inducing symbionts, as shown by many laboratory and field studies. For instance, only five generations were enough for the CI-inducing endosymbiotic bacteria Wolbachia to invade population cages of Drosophila melanogaster (Reynolds and Hoffmann 2002), or of the mosquito Aedes albopictus (Dobson et al. 2002). This bacterium has also been shown to spread rapidly in field populations of different host species (e.g. Turelli and Hoffmann 1995; Kriesner et al. 2013; Bakovic et al. 2018).

Despite the fact that such reproductive manipulation favours the spread of symbionts, stable infection polymorphisms are typical in nature, with some populations being fully infected, others fully uninfected or infected with a different symbiont strain, and others harbouring intermediate symbiont frequencies (e.g. Vavre et al. 2002; Keller et al. 2004; Zhang et al. 2013b; Hamm et al. 2014). This infection polymorphism may be associated with variation in the level of $\mathrm{CI}$, the rate of maternal transmission and the relative fecundity of infected females compared with uninfected ones, which determines the threshold at which a given CI-inducing symbiont can invade a population (Hoffmann et al. 1990; Turelli and Hoffmann 1995). Moreover, variability in infection frequencies between and within regions indicates benefits and costs of infection that vary across temporal and spatial gradients (e.g. Weeks et al. 2002; Oliver et al. 2014; Cass et al. 2016). However, the factors responsible for such variability remain largely elusive. In particular, the relative importance of environmental heterogeneity (e.g. Barton and Turelli 2011; Hancock and Godfray 2012; Schmidt et al. 2017), host diversity and biotic interactions (e.g. within-host interaction with other pathogens or parasites; reviewed in Oliver et al. 2014; Hopkins et al. 2017) in the maintenance of symbiont diversity remains poorly understood.

Laboratory studies may allow to disentangle the effect of the environment and of the host genetic background on symbiont diversity. However, drift and lab adaptation can also deeply impact natural variation. While this has been repeatedly demonstrated regarding nuclear variation (e.g. Hoffmann et al. 2001; Fragata et al. 2014; Francuski et al. 2014; Hoffmann and Ross 2018), few studies have analyzed how laboratory acclimation affects symbiont diversity. Spider mites are good candidates to investigate potential changes in infection polymorphism under laboratory conditions, as they often carry several endosymbiotic bacteria, usually maternally-inherited, with variable prevalence among natural populations. Among them, Wolbachia is the most prevalent (e.g. Liu et al. 2006; Gotoh et al. 2007b; Zhang et al. 2013b; Zhang et al. 2016; Zélé et al. 2018a) and induces variable levels of CI, ranging from no CI to complete CI (Vala et al. 2002; Gotoh et al. 2007b; Xie et al.
2011; Suh et al. 2015). In some cases, in spider mites as in other haplodiploid species, CI involves a loss of the paternal set of chromosomes and diploid zygotes arising from incompatible matings may survive as haploid males (Male development-MD-CI; Perrot-Minnot et al. 2002; Gotoh et al. 2003). In most cases, however, fertilized eggs from incompatible crosses fail to hatch as in diploid species, which leads to embryonic mortality of the females only (Female mortality-FM-CI; Breeuwer 1997; Perrot-Minnot et al. 2002; Vala et al. 2002; Gotoh et al. 2003; Suh et al. 2015). Population-specific fitness effects of Wolbachia on spider mite life-history traits have also been reported, with costs (Perrot-Minnot et al. 2002; Suh et al. 2015), no effect (Breeuwer 1997; Perrot-Minnot et al. 2002; Vala et al. 2002; Gotoh et al. 2007b), or benefits (Vala et al. 2002; Gotoh et al. 2007b; Xie et al. 2011) on spider mite fecundity, but also variable effects on longevity and development time (Xie et al. 2011). Note, however, that none of these studies (with the exception of Gotoh et al. 2007b) tested for coinfection with other endosymbionts, which may have confounding effects. Indeed, herbivorous spider mites are often (co-) infected with Cardinium (Liu et al. 2006; Ros et al. 2012; Zhang et al. 2016), which can also cause FM-CI (Gotoh et al. 2007a; Ros and Breeuwer 2009; Xie et al. 2010; Zhu et al. 2012) without clear effects on other spider mite life-history traits reported to date (but see Zhao et al. 2013a; Zhao et al. 2013b; for WolbachiaCardinium coinfections); and occasionally with Rickettsia (e.g. Zhang et al. 2016; Zélé et al. 2018a) or Spiroplasma (e.g. Enigl and Schausberger 2007; Staudacher et al. 2017), whose effects in spider mites are still unknown.

Here, we analyzed the temporal changes occurring in the prevalence and composition of endosymbionts after transferring spider mite populations from the field to the laboratory. We observed very rapid changes in symbiont diversity, with an apparent loss of Rickettsia and Cardinium, while Wolbachia apparently reached fixation or was lost, after only 6 months ( 15 generations) of laboratory rearing. To understand fixation of Wolbachia, we measured its effects on spider mite life-history traits and the level of CI it induces in each fully infected population. Then, we used these data to parametrize a theoretical model for the invasion process of CIinducing symbionts in haplodiploids. Finally, we discuss the potential factors that may explain the maintenance of symbiont diversity in the field compared with the laboratory.

\section{Materials and methods}

\section{Spider mite populations and rearing}

Sixteen populations of Tetranychid mites were collected from September to December 2013 in the region of Lisbon, 


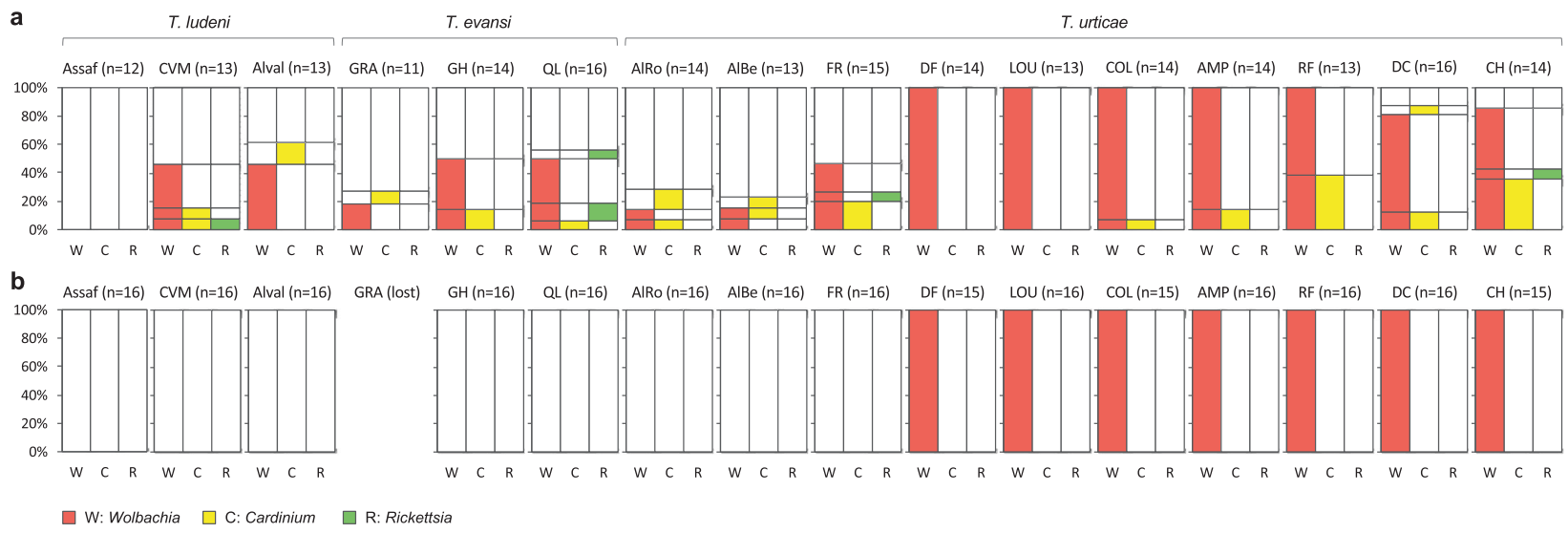

Fig. 1 Changes in endosymbiont infection frequency in each spider mite population following laboratory rearing. Each box represents a population, and within each graph, columns represent the infection status by W: Wolbachia (red cells); C: Cardinium (yellow cells); and R: Rickettsia (green cells) following a 0-3 months, and b 6 months of

laboratory rearing after collection in the field. White cells represent uninfected individuals. Coinfections within the same individuals are indicated by more than one shaded region on the same horizontal plane.

and adult spider mite females from all populations were subsequently individually analyzed for species identification and for the presence of reproductive manipulators (Zélé et al. 2018a). Three of these populations (Assaf, CVM and Alval) belonged to Tetranychus ludeni, three to T. evansi (GRA, GH and QL), and ten to the red form of T. urticae (AlRo, AlBe, FR, DF, LOU, COL, AMP, RF, DC and CH). The prevalence of five maternally-inherited endosymbiotic bacteria was previously estimated using genus-specific PCRs on 11-16 individual females per population (Zélé et al. 2018a). While Wolbachia, Cardinium and Rickettsia infection frequencies varied across populations (Fig. 1a), Arsenophonus and Spiroplasma were absent in all populations. These populations started with variable numbers of foundresses (AlBe: 25; FR: 30; AMP: 65; $\mathrm{CH}$ and GH: 80; COL: 100; Alval: 160; AlRo: 200; LOU and CVM: 300; DC: 400; DF, RF and QL: 500; Assaf: 600). They were then maintained in the laboratory under standard conditions $\left(25 \pm 2{ }^{\circ} \mathrm{C}, 60 \% \mathrm{RH}, 16 / 8 \mathrm{~h} \mathrm{~L} / \mathrm{D}\right)$ at very high numbers (ca. 500-1000 females per cage) in insect-proof cages containing either bean cv. Contender seedlings (obtained from Germisem, Oliveira do Hospital, Portugal) for T. urticae and T. ludeni, or tomato cv. Money Maker seedlings (obtained from Mr Fothergill's Seeds, Kentford, UK) for the solanaceae specialist $T$. evansi.

\section{Screening for infection by endosymbionts and Wolbachia strain identification following laboratory rearing}

Six months after collection from the field (ca. 15 generations), infection by Wolbachia, Cardinium and Rickettsia was checked anew using 15-16 individual females per population (except for the population GRA that was lost during laboratory rearing) using the multiplex PCR described in Zélé et al. (2018c). Subsequently, pools of 100 female per population were also checked for infection by these endosymbionts roughly $6,12,18$ and 24 months after collection from the field (Fig. S1). Previous sensitivity tests revealed that multiple symbionts can be detected in a single pool, even at low infection frequencies (up to 1/100 infected females; Zélé et al. 2018a). Finally, as the wsp gene was identical for all Wolbachia infecting these populations (Zélé et al. 2018a), we characterized the Wolbachia infections remaining in laboratory cultures 6 months after collection using a multilocus sequence typing (MLST; Baldo et al. 2006). MLST gene sequences were amplified from DNA extracted from a pool of 100 females per population using standard primers and PCR protocols (Baldo et al. 2006; Zélé et al. 2018a). Chromatograms were checked manually using MEGA version 5.1 beta (Tamura et al. 2011) and we found no evidence for multiple infections within populations (as indicated by the absence of multiple peaks). All MLST sequences were then compared with entries in the PubMLST Wolbachia MLST database (available at http://www.pubmlst.org/wolbachia/) and novel sequences were submitted to the database curators for inclusion as new alleles. Each unique combination of MLST sequences was designated as an isolate, submitted to the PubMLST database, and assigned a unique ID number. Isolates with fivelocus profiles that did not match an existing strain type were assigned a new strain type (Baldo et al. 2006).

\section{Antibiotic treatments}

Roughly 3 months after collection from the field, a tetracycline solution $(0.1 \%, \mathrm{w} / \mathrm{v})$ was used to treat mites $(n=30$ adult females initially) from each population for three 
successive generations (Breeuwer 1997) to obtain uninfected populations. During the treatment, mites were maintained in petri dishes containing bean (or tomato for $T$. evansi) leaf fragments placed on cotton with the antibiotic solution. At each generation, 50 adult mated daughters were transferred to a new petri dish containing fresh leaf fragments and solution. At the third generation after treatment, 14 individual females and a pool of 100 females per population were checked by PCR to confirm that they were uninfected. These populations were maintained in a massrearing environment without antibiotics for a minimum of five generations before performing experiments, to avoid potential side effects of antibiotic treatment (e.g. Ballard and Melvin 2007; Zeh et al. 2012).

\section{Experiment 1: effects of Wolbachia on T. urticae life- history traits and $\mathrm{Cl}$ induction}

To test the effects of Wolbachia in each population that was still infected 6 months after field collection (all from $T$. urticae), the four possible crosses between Tetracyclinetreated (T) and -untreated (W, Wolbachia-infected) females and males were performed (i.e. $\mathrm{T} \times \mathrm{T}, \mathrm{T} \times \mathrm{W}, \mathrm{W} \times \mathrm{T}$ and $\mathrm{W} \times \mathrm{W}$, female $\times$ male crosses). An additional population (FR), fully uninfected (U) by Wolbachia 6 months after field collection, was also included as a control for the effect of the tetracycline treatment. Roughly 2 weeks prior to the experiment, age cohorts were created for each population by collecting ca. 100 females from each mass culture, allowing them to lay eggs during 5 days on detached bean (or tomato) leaves placed on water-soaked cotton. The offspring from these cohorts was used in the experiments.

Two days prior to the onset of this experiment, quiescent virgin females with similar age were randomly collected from each cohort and placed separately on a leaf fragment to allow emergence while remaining virgin. Males were isolated from the same cohort 1 day before the beginning of the experiment to avoid potential sperm depletion. On the first day of the experiment (d0), 10 adult virgin females were placed with 10 males on a $9 \mathrm{~cm}^{2}$ bean leaf disc to allow mites to mate in panmixia. This procedure was chosen to increase potential conflicts over sex ratio between Wolbachia and its female host. Indeed, while Wolbachia always benefits from a higher proportion of daughters (i.e. due to its maternal mode of transmission; Hurst et al. 1996; Werren and Beukeboom 1998), the optimal sex ratio for female spider mites depends on the number of foundresses in a patch, being more male biased as this number increases (Hamilton 1967; Macke et al. 2011).

Three days later (d3), the daily female oviposition was estimated taking into account their daily mortality (daily oviposition per female over 3 days $=$ total number of eggs laid on each leaf disc after 3 days/total number of alive females over the 3 days), and males were discarded. To determine the effect of Wolbachia on spider mite longevity, females were transferred to new leaf discs every 3 days until death and their daily survival was recorded. To determine the type of $\mathrm{CI}$ induced by Wolbachia in this system (i.e. MD-CI and/or FM-CI; Vavre et al. 2000), the number of unhatched eggs and of adult offspring $\left(F_{1}\right.$ females + $F_{1}$ males) obtained over the first 3 days of the experiment were counted 5 and 15 days after removing the parents, respectively ( $\mathrm{d} 8$ and $\mathrm{d} 18$ ). This allowed computing the relative proportions of unhatched eggs (number of unhatched eggs/total number of eggs), dead juveniles ([total number of eggs - number of unhatched eggs - number of $F_{1}$ adults]/total number of eggs), males (number of $F_{1}$ males/total number of eggs), and females (number of $\mathrm{F}_{1}$ females/total number of eggs) in all populations.

Finally, as we found that Wolbachia induces FM-type of CI in all tested populations (cf. "Results") we determined the level of CI induced by Wolbachia, as the proportion of embryonic death of females in incompatible crosses $\left(\mathrm{CI}_{\mathrm{obs}}\right.$ $=$ number of unhatched eggs/[number of $\mathrm{F}_{1}$ females + number of unhatched eggs]). To account for variation in background embryonic mortality (not related to CI and including both sons and daughters embryonic mortality), we used a corrected index of CI (Poinsot et al. 1998; Cattel et al. 2018) calculated as follows: $\mathrm{CI}_{\text {corr }}=\left[\left(\mathrm{CI}_{\mathrm{obs}}-\mathrm{CCM}\right) /\right.$ $(1-\mathrm{CCM})]$, where CCM is the mean embryonic mortality observed in the control crosses (i.e. calculated as $\mathrm{CI}_{\mathrm{obs}}$ ). To control for an effect of infection on the background embryonic mortality, $\mathrm{T} \times \mathrm{T}$ and $\mathrm{W} \times \mathrm{T}$ crosses were used as controls for $\mathrm{T} \times \mathrm{W}$ and $\mathrm{W} \times \mathrm{W}$ crosses, respectively.

The entire experiment was done in three consecutive blocks, each including four replicates of each cross combination for each mite population, except for "DF", for which all replicates were done in the third block, due to contaminations detected in the previous blocks (i.e. these data were discarded).

\section{Experiment 2: $\mathrm{Cl}$ rescue across Wolbachia-infected T. urticae populations}

To test whether Wolbachia infecting one population can rescue the $\mathrm{CI}$ induced by Wolbachia infecting another population, we performed all possible crosses between Wolbachia-infected populations. The experimental procedure was the same than for intra-populations crosses except that 20 adult virgin females were placed individually with one male on a $2 \mathrm{~cm}^{2}$ bean leaf disc. Subsequently, both males and females were discarded and the number of eggs per individual disc was counted. The relative proportions of unhatched eggs, dead juveniles, males, and females were subsequently measured as previously described. To avoid biases arising from low number of eggs in proportion data, all females that laid less than five eggs within the first 3 days of the experiment were removed from statistical analyses (cf. final sample sizes in Table S3). Subsequently, 
$\mathrm{CI}_{\text {corr }}$ was calculated as above, using each intra-population cross as control for a given female population when crossed with males from all other populations.

All experiments were conducted in a growth chamber under standard conditions $\left(25 \pm 2{ }^{\circ} \mathrm{C}, 60 \% \mathrm{RH}, 16 / 8 \mathrm{~h} \mathrm{~L} / \mathrm{D}\right)$.

\section{Statistical analyses}

Analyses were carried out using the R statistical package (v. 3.6.0). The different statistical models built to analyse the phenotypic effects of Wolbachia in both intra- and interpopulation crosses are described in the Supplementary materials, Table S1. The general procedure for building the statistical models was as follows: the status of females and their mates (i.e. treated with tetracycline or not in the first experiment, and the populations the individuals belonged to in the second experiment), were fit as fixed explanatory variables, whereas blocks (and leaf discs for survival analyses) were fit as random explanatory variables.

Survival data (models 1.0-1.8) were analysed using Cox proportional hazards mixed-effect models (coxme, kinship package). Hazard ratios (HR) were obtained from these models as an estimate of the difference between the rates of dying (i.e. the instantaneous rate of change in the $\log$ number of survivors per unit time; Crawley 2007) between the control and the other crosses. All other response variables were analysed using generalized linear mixed models with the glmmTMB procedure (glmmTMB package; Brooks et al. 2017), which allows using a wide range of error distribution that are not implemented in the glmer procedure. Female daily oviposition was analysed with a gamma error distribution with a log link to account for heteroscedasticity (models 2.0-2.8). Proportion data were computed using the function cbind, except for $\mathrm{CI}_{\text {corr }}$ (continuous variable bounded between 0 and 1) for which a "weights" argument was added in the model to account for the number of observations (i.e. number of unhatched eggs + number of adult daughters per disc). Proportion data were subsequently analysed with a binomial error distribution, or with a betabinomial error distribution to account for overdispersed errors (models 3.0-12.0).

Maximal models, including all higher-order interactions, were simplified by sequentially eliminating non-significant terms and interactions to establish a minimal model, and the significance of the explanatory variables was established using chi-squared tests (Crawley 2007). The significant $X^{2}$ values given in the text are for the minimal model (Crawley 2007). When the variable "population" was found to interact significantly with other variables, each population was analysed separately to determine the effect of the status of both females and males, as well as their interactions. When a significant interaction between these explanatory variables was found, a posteriori orthogonal contrasts
(Crawley 2007) between crosses ("W $\times \mathrm{W}$ ", "W $\times \mathrm{T}$ ", "T $\times$ $\mathrm{W}$ " and " $\mathrm{T} \times \mathrm{T}$ ") were carried out by aggregating factor levels together and by testing the fit of the simplified model using ANOVA. In the case of $\mathrm{CI}_{\text {corr }}$, compatible and incompatible crosses were analysed separately to determine differences between populations.

\section{Modelling Wolbachia invasion under laboratory conditions}

To predict Wolbachia invasion in each population that was fully infected 6 months after collection, we used the data obtained for the phenotypic effects of Wolbachia to parameterize a mathematical model for FM-type CI (cf. "Results") developed by Vavre et al. (2000). This model allows estimating the value of the unstable equilibrium (i.e. the threshold for infection rates above which Wolbachia is expected to reach fixation, and below which it is predicted to go extinct; Hoffmann et al. 1990). The parameters of this model are the relative fecundity of infected versus uninfected females $(F$; here this parameter was also weighted by the effect of Wolbachia on the female survival, so $\mathrm{F}=$ mean daily oviposition of infected females [incl. $\mathrm{W} \times \mathrm{W}$ and $\mathrm{W} \times \mathrm{T}$ crosses] over 3 days/mean daily oviposition of uninfected females [incl. $\mathrm{T} \times \mathrm{W}$ and $\mathrm{T} \times \mathrm{T}$ crosses] over 3 days/hazard ratio of infection in females), the proportion of eggs that escape $\mathrm{CI}$ in the incompatible cross $(\mathrm{H}$; i.e. the reverse of the $\mathrm{CI}$ level, so here $\left.\mathrm{H}=1-\left(\mathrm{CI}_{\text {corr }} / 100\right)\right)$, and the proportion of uninfected eggs produced by infected females ( $\mu$; i.e. the reverse of the transmission rate). We assumed perfect maternal transmission as only a transmission rate of $100 \%$ may explain an observed infection frequency of $100 \%$ in females when CI is incomplete. Nevertheless, to account for potential inaccuracy of observed infection frequencies, we estimated the minimum transmission rate that can explain the maintenance of Wolbachia in each population (Table S5).

\section{Results}

\section{Changes in endosymbiont prevalence under laboratory conditions}

The screen for endosymbiont infection following 6 months of laboratory rearing (ca. 15 generations) revealed a drastic change in symbiont prevalence after field collection (Fig. 1a and described in Zélé et al. 2018a). Indeed, neither Cardinium nor Rickettsia were detected in any of the populations tested (prevalence $<11 \%$ with $95 \%$ confidence intervals; Jeffreys interval recommended for small $n$ by Brown et al. 2001), whereas all females were found infected by Wolbachia in seven T. urticae populations (prevalence $>88-89 \%$ with $95 \%$ confidence intervals), and none of them in eight 
populations, belonging to T. urticae, T. evansi and T. ludeni (prevalence $<11 \%$ with $95 \%$ confidence intervals; Fig. 1b). Moreover, diagnostic PCRs performed on pools of 100 females 6, 12, 18 and 24 months after field collection (Fig. $\mathrm{S} 1$ ) confirmed the loss (prevalence $<1 \%$ ) of endosymbionts in these populations. In general, there is a good correlation between the symbiont frequency in the original population and the probability of infection loss or fixation. Indeed, Wolbachia was lost in the populations in which its initial frequency was lower than $50 \%$, while it reached fixation in the other populations.

\section{Wolbachia diversity in the laboratory}

The MLST sequences were the same for all Wolbachia that reached fixation in $T$. urticae populations. This confirms the results previously obtained using the wsp gene (i.e. only one wsp sequence was found across all populations, GenBank: DQ910771; Zélé et al. 2018a) although we cannot rule out that diversity existed in field collected samples, and that the same (or a similar) Wolbachia variant reached fixation in all populations under our laboratory conditions. Most sequences found were already present in the PubMLST database (gatB: allele 9; $\operatorname{coxA}$ : allele 38; $h c p A$ : allele 143, and ftsZ: allele 23), but we identified a new allele for $f b p A$ : the allele 444 , which presents one SNP with the existing allele 4. Consequently, we defined a new strain of Wolbachia, ST491, which is very similar to the strain ST219 belonging to supergroup B that was found in China by Zhang et al. (2013a).

\section{Experiment 1: effects of Wolbachia on T. urticae life- history traits and $\mathrm{Cl}$ induction}

\section{Effects of Wolbachia on spider mite longevity}

As all symbionts were lost in T. evansi and T. ludeni, the following results were obtained only in the $T$. urticae populations in which Wolbachia reached fixation in the laboratory. Daily female survival was significantly affected by the status (treated with tetracycline or not) of both the females and their mates, but in a population-specific manner (model 1.0 in Table S1, see also Table S2 for log HRs and the significance of all fixed effects and their interactions; Fig. S2 for survival curves). Indeed, the independent analysis of each population showed that the tetracycline treatment did not affect longevity in the populations AMP, DF and the uninfected control FR (model 1.1-1.3), while in $\mathrm{CH}$ and COL Wolbachia-infected females had a ca. 1.5 and 1.3 times shorter lifespan than uninfected females, respectively (model 1.4, $X^{2}{ }_{1}=16.34, p<0.0001$, and model $1.5, X^{2}{ }_{1}=$ $6.40, p=0.01$, respectively). In addition, females mated with a Wolbachia-infected male survived 1.3 and 1.6 times less than those mated with an uninfected male in COL and LOU, respectively (model $1.5, X^{2}{ }_{1}=5.08, p=0.02$, and model 1.6, $X^{2}{ }_{1}=17.81, p<0.0001$, respectively). Conversely, females mated with a Wolbachia-infected male survived 0.8 and 0.7 times longer than those mated with an uninfected male in DC and RF (model 1.7, $X^{2}{ }_{1}=5.04, p=$ 0.02 , and model $1.8, X^{2}{ }_{1}=11.98, p=0.0005$, respectively).

\section{Effects of Wolbachia on spider mite fecundity}

The analysis of daily female oviposition over 3 days revealed no significant 3-way interaction between populations, female and male infection status (model 2.0, see Table S2 for the significance of all fixed effects and their interactions). Sequential removal of non-significant factors (including their interactions) from the model unveiled no significant interaction between female and male infection status and between population and male infection status, nor a significant effect of male infection status. However, a significant interaction between population and female infection status was found (Fig. 2). The independent analysis of each population further revealed variable effects of Wolbachia infection in females depending on the population: decreased oviposition by $0.93 \pm 0.45$ in AMP (model

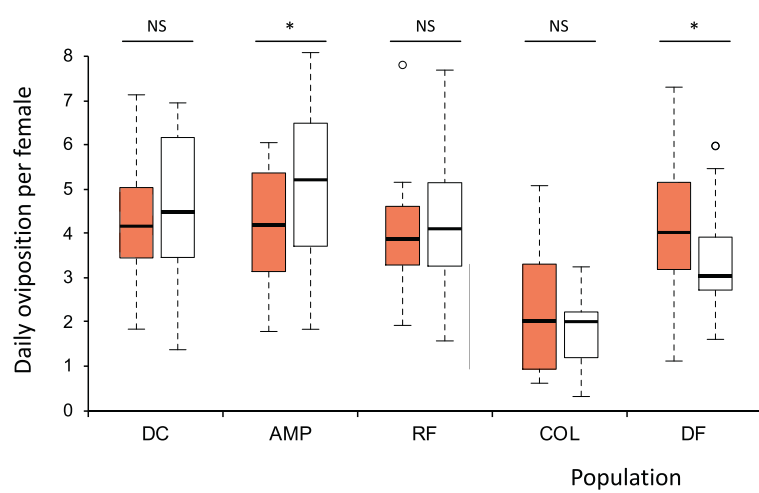

Fig. 2 Wolbachia effects on oviposition of $T$. urticae females. Orange boxes: Wolbachia-infected females, white boxes: tetracyclinetreated females. The statistical significances are given above bars: ${ }^{*} p<$

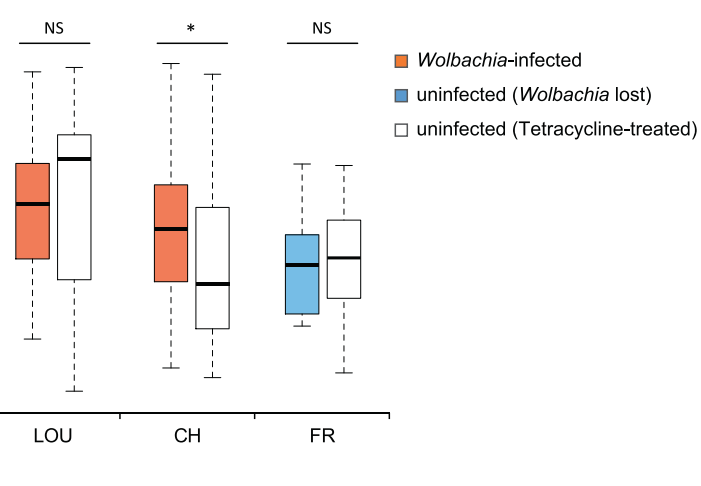

0.05; ns not significantly different at the $5 \%$ level. The population FR (blue box) lost Wolbachia in the laboratory and is used here as control for the tetracycline treatment. 


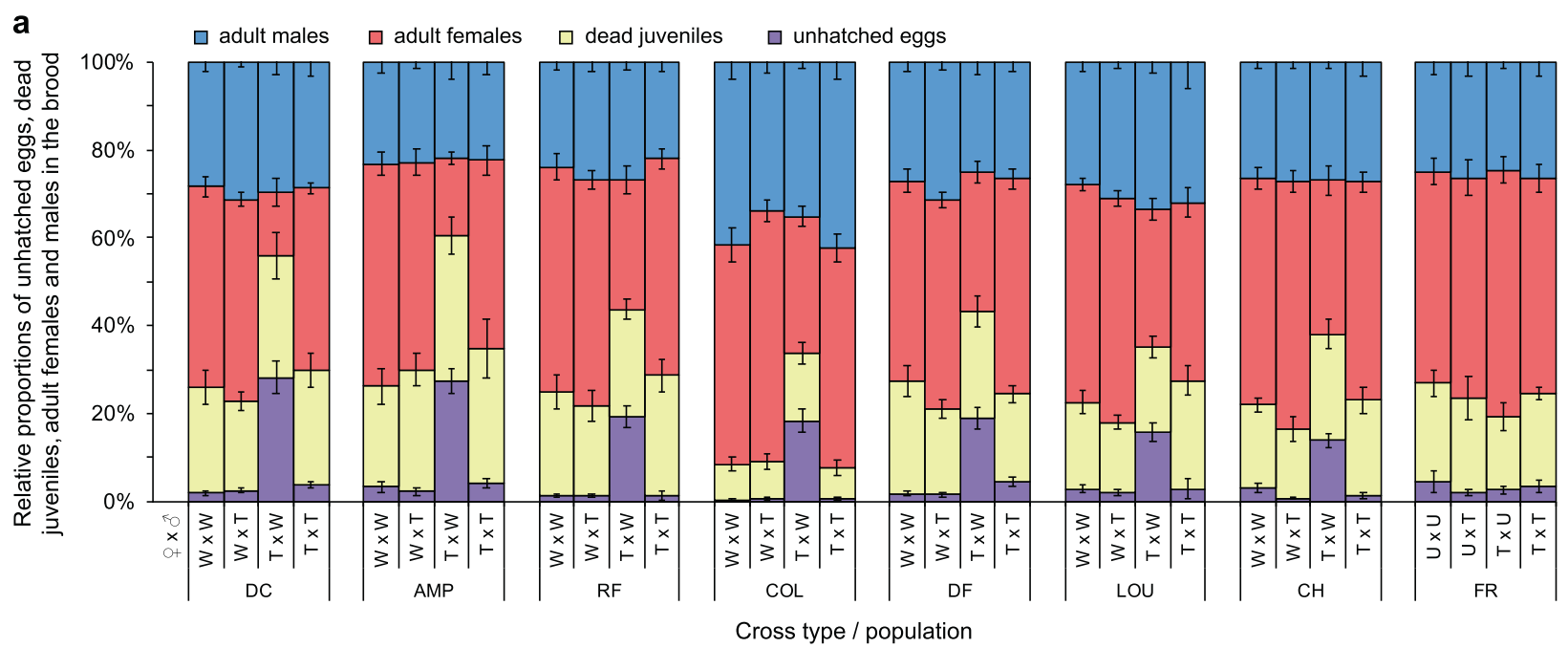

b

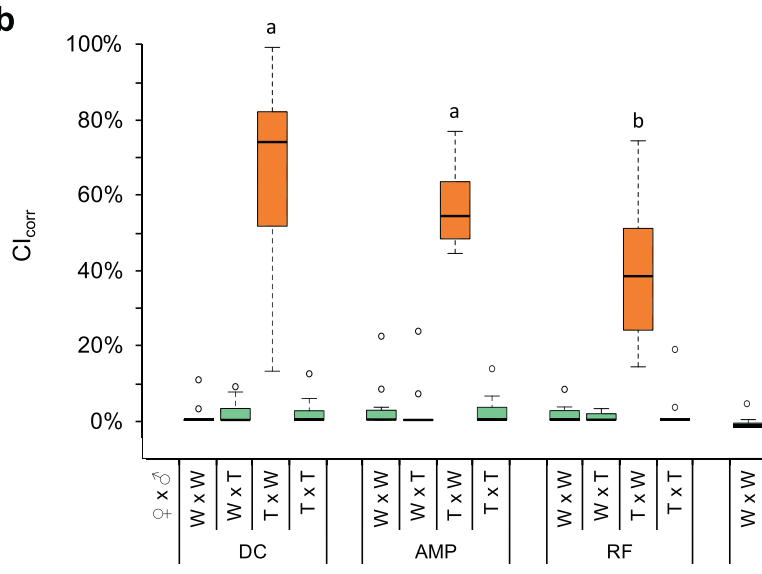

$\square$ tetracycline-treated females $x$ untreated males $\square$ all other crosses
Fig. 3 Summary of the development of T. urticae eggs and cytoplasmic incompatibility (CI) levels in intra-population crosses between Wolbachia-infected and uninfected mites. a Relative proportions of unhatched eggs (purple bars), dead juveniles (yellow bars), adult females (red bars) and adult males (blue bars) for each type possible cross. Bar plots represent means \pm s.e. (values provided in Table S2). T: tetracycline-treated; W: Wolbachia-infected; U: naturally Wolbachia-uninfected. The population FR lost Wolbachia in the

$\left.2.1, X^{2}=5.84, p=0.02\right)$, increased oviposition by $0.77 \pm$ 0.36 in DF (model $2.2, X^{2}{ }_{1}=4.31, p=0.04$ ) and by $0.97 \pm$ 0.54 in $\mathrm{CH}$ (model 2.3, $X^{2}{ }_{1}=6.41, p=0.01$ ), but no significant effect of Wolbachia infection in the other populations, including the control population FR (models 2.4-2.8, DC: $X^{2}{ }_{1}=0.40, p=0.52$, RF: $X^{2}{ }_{1}=0.54, p=0.46$, COL: $X^{2}{ }_{1}=0.68, p=0.41$, LOU: $X^{2}{ }_{1}=0.15, p=0.70$, FR: $X^{2}{ }_{1}$ $=0.36, p=0.55$ ).

\section{Effects of Wolbachia on offspring development}

Overall, the relative proportion of unhatched eggs varied according to the tested population and the infection status of both males and females (model 3.0, see Table S2 for the significance of all fixed effects and their interactions; Fig. laboratory and is used as control for tetracycline treatment. b Boxplot of CI-related mortality estimated using the $\mathrm{CI}_{\text {corr }}$ index, which removes the basal embryonic mortality (estimated in control crosses). Identical or absent superscripts indicate non-significant differences at the 5\% level among populations for crosses between tetracycline-treated females and untreated males ("T $\times \mathrm{W} / \mathrm{U}$ "; orange boxes). No significant differences were found between all other crosses ("T $\times \mathrm{T}$ ", "U/ $\mathrm{W} \times \mathrm{T}$ ", "U/W $\times \mathrm{U} / \mathrm{W} "$; green boxes).

3a). Indeed, in all populations, except in the control FR, the proportion of unhatched eggs was higher in crosses between uninfected females mated with infected males than in other crosses, which indicates the induction of CI by Wolbachia (models 3.1-3.8; see Table S2 for the results of the contrasts analyses). The relative proportion of females also varied according to the tested population and the infection status of both males and females (model 5.0, Table S2), and in all populations, except in the control FR, the proportion of females was lower in incompatible than in compatible crosses (models 5.1-5.8; Table S2). Conversely, the relative proportion of males only differed between populations independently of Wolbachia infection in males and females (model 6.0; Table S2). As the increased proportion of unhatched eggs in incompatible crosses led to a decrease in 


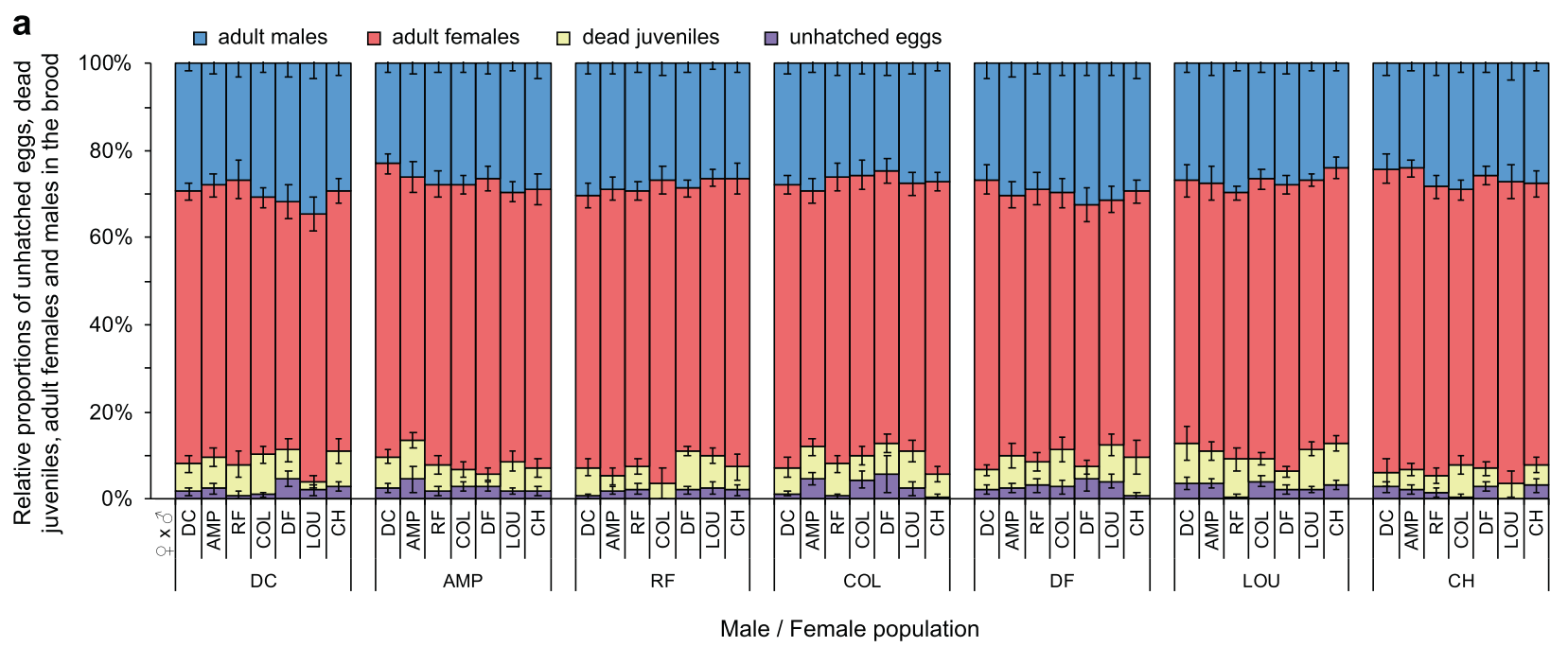

b

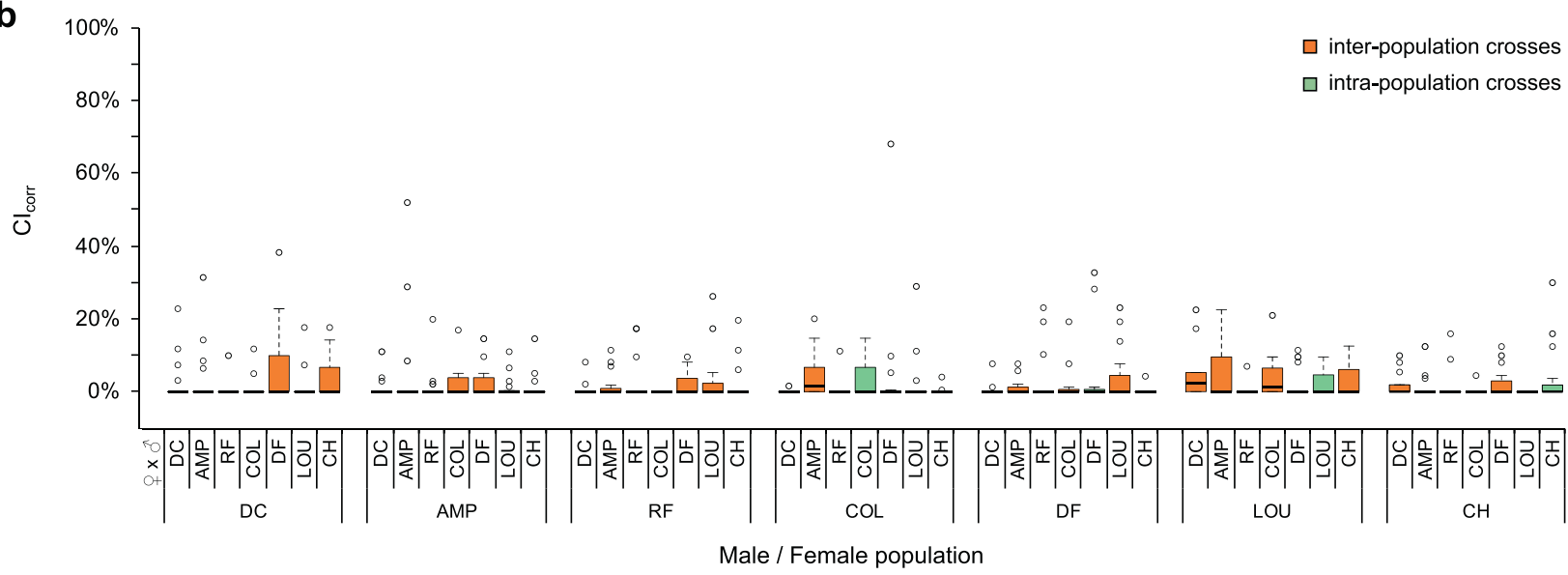

Fig. 4 Summary of the development of T. urticae eggs and cytoplasmic incompatibility (CI) levels in inter-population crosses using Wolbachia-infected mites. a Relative proportions of unhatched eggs (purple bars), dead juveniles (yellow bars), adult females (red bars) and adult males (blue bars) for each type possible cross. Bar plots represent means \pm s.e. (values provided in Table S3). b Boxplot of CIrelated mortality estimated using the $\mathrm{CI}_{\text {corr }}$ index, which removes the basal embryonic mortality (estimated in control crosses). No significant differences were found among crosses (green boxes: intrapopulation crosses; orange boxes: inter-population crosses). the production of females but not of males, these results indicate that $\mathrm{CI}$ induced by Wolbachia does not lead to haploidization of fertilized eggs (MD-type of CI) but to female early mortality (FM-type of CI) in all populations. Finally, the relative proportion of dead juveniles differed between populations and was affected by Wolbachia infection in females, with an overall decreased juvenile mortality of ca. $3 \%$ in the offspring of infected females, but no significant interaction was found (model 4.0; Table S2).

\section{$\mathrm{Cl}$ level induced by Wolbachia in each population}

Females were produced in all incompatible crosses showing that CI was incomplete. Moreover, the analysis of the level of $\mathrm{CI}_{\text {corr }}$ in incompatible crosses showed a significant interaction between the tested population and the infection status of both males and females (model 7.0, Table S2).
While no difference was found between compatible crosses of all populations (model 7.1, Table S2), a significant difference was found between populations for incompatible crosses (model 7.2, Fig. 3b and Table S2). The contrast analysis revealed no significant difference between AMP and DC $\left(X^{2}{ }_{1}=1.74, p=0.19\right)$ and among RF, COL, DF, LOU and $\mathrm{CH}\left(X^{2}{ }_{4}=3.72, p=0.45\right)$, but a significantly lower level of $\mathrm{CI}$ in the latter than in the former group of populations (on average $33 \%$ and $61 \%$, respectively; $X^{2}{ }_{1}=$ 38.37, $p<0.0001)$. All infected populations differed significantly from the control FR $\left(X^{2}{ }_{1}=68.90, p<0.0001\right)$.

\section{Experiment 2: $\mathrm{Cl}$ rescue across Wolbachia-infected $T$. urticae populations}

The ability of Wolbachia infection in females from each population to rescue CI induced by Wolbachia infection in 

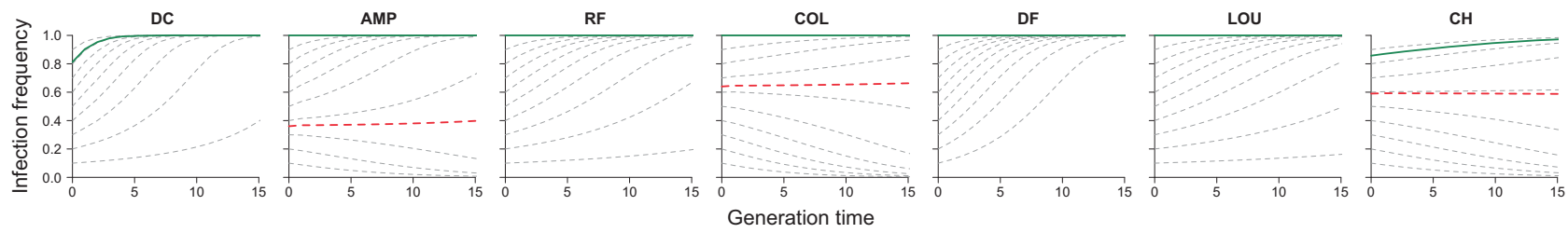

Fig. 5 Expected invasion of Wolbachia based on its phenotypic effects in each population. We used the data obtained for the phenotypic effects of Wolbachia to parametrize the model for each population that fixed the infection under laboratory rearing (parameter values provided in Table S4). Dashed grey lines represent the course of infection frequencies through generations for initial infection frequencies ranging from 0.1 to 0.9 . Green line: course of infection that took place in the laboratory following the prediction of the model; Dashed red line: threshold for invasion.

populations DC, RF, DF and LOU, whatever its initial infection frequency (i.e. unstable equilibrium $<0$ ), as no fecundity and longevity costs associated with infection were detected. For the populations AMP, $\mathrm{COL}$ and $\mathrm{CH}$, the model predicts the existence of an unstable equilibrium above which infection should spread. Due to fitness costs of infection (on oviposition and/or longevity), this unstable equilibrium was relatively high, especially in the populations $\mathrm{COL}$ and $\mathrm{CH}$ in which it was above 50\% (Fig. 5 and Table S4). As the initial frequency of Wolbachia infection in each of these population was above their respective unstable equilibrium, the rapid invasion of Wolbachia observed in the laboratory is in accordance with theoretical predictions.

\section{Discussion}

In a previous study conducted in southwest Europe on 16 natural populations of Tetranychus spider mites, we detected Wolbachia, Cardinium, and Rickettsia with highly variable prevalence (Zélé et al. 2018a). Here, we report a rapid change of the infection status of these populations after only 6 months of laboratory rearing (ca. 15 generations of laboratory evolution), from an apparent loss of Rickettsia and Cardinium to apparent fixation or loss of Wolbachia. In the seven populations where Wolbachia remained (all from T. urticae), we found variable effects of infection on host traits.

\section{Variability in Wolbachia effects and level of $\mathrm{Cl}$}

Wolbachia affected differently the longevity of females from different populations, with either no effect or a cost of infection on survival. Moreover, we found variable effects of mating with Wolbachia-infected males on this trait, with both positive and negative effects, as previously found in $T$. urticae populations in China (Xie et al. 2011). Wolbachia also affected female fecundity differently depending on the population, ranging from no effect to costs or benefits, as in many spider mite populations worldwide (Breeuwer 1997; 
Perrot-Minnot et al. 2002; Vala et al. 2002; Gotoh et al. 2007b; Xie et al. 2011; Suh et al. 2015). These effects, although of relatively low amplitudes may still have important consequences for the invasion dynamics of Wolbachia (e.g. the existence of an invasion threshold when Wolbachia induces a fecundity or a longevity cost, independently of the level of CI it induces; Fig. 5).

The analysis of the proportions of unhatched eggs, daughters and sons in the brood revealed that Wolbachia induces a female mortality type of CI (FM-CI; Breeuwer 1997; Vavre et al. 2000) in all populations. However, besides the sex ratio distortion observed in incompatible crosses due to CI, we did not find any effect of Wolbachia on the offspring sex ratio in compatible crosses. This suggests that sex ratio distortion induced by Wolbachia in absence of CI, as observed by Vala et al. (2003), is not a common feature of Wolbachia in spider mites.

Finally, we found that the level of CI induced by Wolbachia also varies depending on the population (ca. $33 \%$ in the populations RF, COL, DF, LOU and $\mathrm{CH}$, and ca. $61 \%$ in AMP and DC), albeit Wolbachia wsp (Zélé et al. 2018a) and MLST sequences at the time of the experiment did not differ among populations. Such variability of FM-CI levels induced by Wolbachia, without clear association with different Wolbachia wsp sequences, has been previously reported in spider mites (Vala et al. 2002; Gotoh et al. 2003; Gotoh et al. 2007b; Xie et al. 2011; Suh et al. 2015). However, although the use of wsp and of the MLST approach is a standard in the community of Wolbachia researchers, these genes may not be particularly suited to discriminate between closely-related strains (Ishmael et al. 2009; Atyame et al. 2011; Conner et al. 2017), or to accurately reflect the properties of a Wolbachia strain (Bleidorn and Gerth 2018), including different level of CI induction (Hamm et al. 2014; Kaur et al. 2017). In particular, genes responsible for $\mathrm{CI}$ induction (the $\operatorname{cidA}-\operatorname{cidB}$ or $\operatorname{cif} A-\operatorname{cif} B$, and and $\operatorname{cin} A-\operatorname{cin} B$ operons) have recently been identified in different Wolbachia strains infecting different hosts (Beckmann et al. 2017; LePage et al. 2017; Bonneau et al. 2018; Lindsey et al. 2018). It has been proposed that CI strength could be adjusted via the level of expression of these genes, or the ratio of cifA and cifB transcripts across development (Lindsey et al. 2018). Our populations could thus be infected with different but closely-related Wolbachia strains differing for these genes. Unfortunately, we failed to amplify the cidA and cidB genes of Wolbachia in T. urticae (see Box S1) and future work should focus on sequencing the entire genome of Wolbachia from spider mites to improve our understanding of this system. Still, the absence of sequence divergence among Wolbachia from different populations is in agreement with our finding that all populations were compatible with each other (i.e. full CI rescue between populations). Therefore, variations across $T$. urticae populations in fitness effects and in the strength of reproductive phenotypes may be due to the hosts specific genetic backgrounds as shown in some drosophila species (e.g. Reynolds and Hoffmann 2002; Mercot and Charlat 2004; Cooper et al. 2017), but also in T. urticae (Sun et al. 2016).

\section{Loss or fixation of endosymbionts in the laboratory}

We found contrasting evolutionary dynamics of invasion of Wolbachia across the sixteen populations, with rapid invasion leading to fixation in seven populations, and its loss in all others. Cardinium and Rickettsia were also lost in all populations. Stochastic effects (i.e. random genetic drift) may play an important role in the fate of endosymbionts in the laboratory, especially for low initial infection frequencies or small host population sizes (Jansen et al. 2008; Reuter et al. 2008; Oliver et al. 2014). In this study, founder effects may thus explain the loss of infection in some populations that were started from few individuals (e.g. AlBe and FR), or very low initial symbiont infection frequencies (Fig. 1a). However, most populations were founded with relatively high numbers of individuals, and all were subsequently maintained at very high numbers. Moreover, the deterministic model of Vavre et al. (2000) parameterized with our data predicted a rapid invasion of Wolbachia in all populations in which we could study its effects, even from low or mid initial infection frequencies (e.g. in the populations COL, DF and LOU, and in the populations DC, AMP and RF, respectively). It suggests that the fixation of Wolbachia observed in the laboratory were mostly determined by $\mathrm{CI}$, rather than by the fitness effects of this symbiont and/or by drift.

The spread of CI-inducing symbionts is predicted to be more likely than that of a comparable neutral genetic element, even in the face of an invasion threshold (Jansen et al. 2008). Therefore, the loss of endosymbionts in populations with high population density, and when the initial infection frequency was close to 50\% (e.g. Wolbachia in CVM, Alval, GH and QL, or Cardinium in RF and $\mathrm{CH}$ ), suggests that the lost symbionts did not induce high $\mathrm{CI}$ levels that could compensate for fitness costs (e.g. due to fitness costs of infection, the populations $\mathrm{AMP}, \mathrm{COL}$ and $\mathrm{CH}$ are also expected to lose the infection for an initial infection frequency below 36, 70 and 59\%, respectively; Fig. 5) and/or drift effects. Indeed, not only variability in CI levels is a common feature in spider mites, but several studies have also reported infections by non CI-inducing Wolbachia (Perrot-Minnot et al. 2002; Vala et al. 2002; Gotoh et al. 2003, 2007b; Xie et al. 2011; Suh et al. 2015) and Cardinium (Gotoh et al. 2007a) strains. Moreover, although Wolbachia and Cardinium transmission rates were found to be often close to one in arthropods (e.g. Rasgon and Scott 
2003; Narita et al. 2007; Perlman et al. 2008), this might not be the case for all strains, and in all host species/populations. Unfortunately, the transmission rate of Cardinium, Rickettsia, and of Wolbachia infecting the populations in which they were lost is unknown here.

Hence, although the invasion by Wolbachia can easily be explained by its phenotypic effects on the host, its loss and that of Cardinium and Rickettsia, can be attributed to any factor (e.g. inefficient maternal transmission, absence or low CI induction, high fitness costs, stochastic effects).

\section{What explains the maintenance of symbiont diversity in the field compared with the lab?}

It should be noticed that we did not find an effect of collection date on the probability of infection by Wolbachia in these field populations (Zélé et al. 2018a). Moreover, another field collection of T. urticae populations, conducted 2 years later in the same region in Portugal, shows that the prevalence of the three endosymbionts remained relatively similar (Zélé et al. 2018b). Diversity and polymorphism thus seem stable in field populations. If symbionts in the lab rapidly reached fixation or extinction, then what maintains different prevalence levels between populations in the field and polymorphism within populations? A few, non-exclusive, hypotheses can be put forward.

Different prevalence levels between populations might be explained by spatial variation of environmental conditions in the field, which may impact the effects of endosymbionts on host fitness. For example, temperature is known to affect endosymbiont transmission, their fitness effects on hosts and the strength of reproductive manipulation (e.g. Clancy and Hoffmann 1998; Anbutsu et al. 2008; Carrington et al. 2010; Bordenstein and Bordenstein 2011; Ross et al. 2017b). In line with this, Wolbachia prevalence varies with temperature in the field (e.g. Toju and Fukatsu 2011; Sumi et al. 2017; Ferguson et al. 2018), and, in spider mites, a field study shows that Wolbachia prevalence increases with temperature (e.g. Zhu et al. 2018), but a too high temperature cures mites from this symbiont (e.g. Van Opijnen and Breeuwer 1999). Spatial variation in other environmental factors such as host nutrition (e.g. Clancy and Hoffmann 1998), including the host plant of herbivorous arthropods (reviewed in Frago et al. 2012), and/ or the presence of host pathogens or natural enemies (reviewed in Oliver et al. 2014; Hopkins et al. 2017), may affect the prevalence of symbionts and explain differences between populations. Similarly, temporal (seasonal and/or circadian) variations in all these factors may lead to temporal variations in endosymbiont prevalence within populations and, hence, may explain the maintenance of infection polymorphism at the population level.
Another possible means to maintain variation in prevalence levels between populations is spatial structure of different host genotypes (i.e. limited gene flow between populations), which may be more or less pervasive to CI or other fitness effect of the symbionts (see above). Many studies have shown the existence of population structure in spider mites (reviewed in Sousa et al. 2019). Hence, migrations among populations with variable infection prevalence should blur differences in prevalence levels between populations. However, they may also allow maintaining infection polymorphism within populations. Indeed, several models predict that (positive) frequencydependent selection on CI prevents stable coexistence of infected and uninfected hosts in a panmictic population, but enables it in structured populations, in which migration rate falls below a critical value (reviewed in Engelstadter and Telschow 2009).

Finally, infection polymorphism within field populations may be maintained by horizontal transfers of symbiont between hosts from different populations or species. Evidences of horizontal transfers come from incongruences between phylogenies of host and symbionts in spider mites (e.g. Yu et al. 2011; Ros et al. 2012), as in many other arthropod hosts (e.g. Vavre et al. 1999; Raychoudhury et al. 2009; Ahmed et al. 2016; Conner et al. 2017). If such horizontal transfers are frequent enough in field populations, they could play a role in the infection dynamics of the symbionts and allow the maintenance of some symbionts at low frequency.

\section{Future directions}

We observed a rapid loss of endosymbionts diversity following colonization in a laboratory environment. Such lability of endosymbionts can be particularly useful to develop and experimentally test theoretical models of symbiont invasion. However, such laboratory studies may also not reflect the processes at play in the field, thereby hampering a good understanding of host-symbiont interactions.

Important efforts have recently been developed to understand the effect of the transition from the laboratory to the field on the dynamic of Wolbachia within mosquito populations due to its implication for disease control (e.g. Hoffmann et al. 2014; Nguyen et al. 2015). In particular, our observations highlight the relevance of the new methods that are currently developed to minimize laboratory adaptation and, hence, to increase the relevance of laboratory experiments for the understanding of natural populations (Leftwich et al. 2016; Ross et al. 2017a).

Although some studies report rapid genetic changes in arthropods during a transition from the field to the laboratory (e.g. Hoffmann et al. 2001; Fragata et al. 2014; Francuski et al. 2014), changes in symbiotic communities are 
still largely understudied. This is at odds with the relevance they may have for implementing existing studies of host adaptation to novel environment (e.g. Matos et al. 2015; Fragata et al. 2016; Hoffmann and Ross 2018). Whether the loss or fixation of particular symbionts (strains or species) under laboratory conditions is adaptive for the host, or whether it is a by-product of the host environment on the symbiotic community, remains elusive.

\section{Data availability}

Full datasets have been deposited in the Dryad data repository (https://doi.org/10.5061/dryad.pk0p2ngjg).

Acknowledgements We are grateful to Joaquin Calatayud, Salomé Clémente, Diogo Godinho and Leonor Rodrigues for their help in collecting data from the intra-population crosses; to André Alves, Catarina Bota, Jéssica Paulo, José Leitão, Andreia Oliveira and Luís Silva for the inter-population crosses. We also thank Patrick Makoundou for his attempts to amplify cidA and $c i d B$ gene fragments from $T$. urticae. Finally, we thank Olivier Duron, Inês Fragata, Michael Turelli and Filipa Vala for useful discussions and suggestions. This work was funded by an FCT-ANR project (FCT-ANR//BIAEVF/0013/2012) to SM and Isabelle Olivieri, and by an FCT-Tubitak project (FCT-TUBITAK/0001/2014) to SM and Ibrahim Cakmak. FZ was funded through an FCT Post-Doc fellowship (SFRH/BPD/ 125020/2016). Funding agencies did not participate in the design or analysis of experiments.

Author contributions Designed the project: FZ and SM, with discussions with MM, MW and FV. Designed experiments: FZ, SM; population maintenance: IS; molecular analyses: FZ, MW; performed the experiments: FZ and IS; statistical analyses and model application: FZ; paper writing: FZ, FV and SM with input from all authors. All authors read and approved the final version of the manuscript.

\section{Compliance with ethical standards}

Conflict of interest The authors declare that they have no conflict of interest.

Publisher's note Springer Nature remains neutral with regard to jurisdictional claims in published maps and institutional affiliations.

\section{References}

Ahmed MZ, Breinholt JW, Kawahara AY (2016) Evidence for common horizontal transmission of Wolbachia among butterflies and moths. BMC Evol Biol 16:118

Anbutsu H, Goto S, Fukatsu T (2008) High and low temperatures differently affect infection density and vertical transmission of male-killing Spiroplasma symbionts in Drosophila hosts. Appl Environ Microbiol 74(19):6053-6059

Atyame CM, Delsuc F, Pasteur N, Weill M, Duron O (2011) Diversification of Wolbachia endosymbiont in the Culex pipiens mosquito. Mol Biol Evol 28(10):2761-2772

Bakovic V, Schebeck M, Telschow A, Stauffer C, Schuler H (2018) Spatial spread of Wolbachia in Rhagoletis cerasi populations. Biol Lett 14(5):pii: 20180161
Baldo L, Hotopp JCD, Jolley KA, Bordenstein SR, Biber SA, Choudhury RR et al. (2006) Multilocus sequence typing system for the endosymbiont Wolbachia pipientis. Appl Environ Microbiol 72(11):7098-7110

Ballard JWO, Melvin RG (2007) Tetracycline treatment influences mitochondrial metabolism and mtDNA density two generations after treatment in Drosophila. Insect Mol Biol 16(6):799-802

Barton NH, Turelli M (2011) Spatial waves of advance with bistable dynamics: cytoplasmic and genetic analogues of allee effects. Am Nat 178(3):E48-E75

Beckmann JF, Ronau JA, Hochstrasser M (2017) A Wolbachia deubiquitylating enzyme induces cytoplasmic incompatibility. Nat Microbiol 2(5):17007

Bleidorn C, Gerth M (2018) A critical re-evaluation of multilocus sequence typing (MLST) efforts in Wolbachia. FEMS Microbiol Ecol 94(1):fix 163

Bonneau M, Atyame C, Beji M, Justy F, Cohen-Gonsaud M, Sicard M et al. (2018) Culex pipiens crossing type diversity is governed by an amplified and polymorphic operon of Wolbachia. Nat Commun 9:1491

Bordenstein SR, Bordenstein SR (2011) Temperature affects the tripartite interactions between bacteriophage WO, Wolbachia, and cytoplasmic incompatibility. PLoS ONE 6(12):11

Breeuwer JAJ (1997) Wolbachia and cytoplasmic incompatibility in the spider mites Tetranychus urticae and T. turkestani. Heredity 79:41-47

Brooks ME, Kristensen K, van Benthem KJ, Magnusson A, Berg CW, Nielsen A et al. (2017) glmmTMB balances speed and flexibility among packages for zero-inflated generalized linear mixed modeling. R J 9(2):378-400

Brown LD, Cai TT, DasGupta A (2001) Interval estimation for a binomial proportion. Stat Sci 16(2):101-117

Carrington LB, Hoffmann AA, Weeks AR (2010) Monitoring longterm evolutionary changes following Wolbachia introduction into a novel host: the Wolbachia popcorn infection in Drosophila simulans. Proc R Soc B 277(1690):2059-2068

Cass BN, Himler AG, Bondy EC, Bergen JE, Fung SK, Kelly SE et al. (2016) Conditional fitness benefits of the Rickettsia bacterial symbiont in an insect pest. Oecologia 180(1):169-179

Cattel J, Nikolouli K, Andrieux T, Martinez J, Jiggins F, Charlat S et al. (2018) Back and forth Wolbachia transfers reveal efficient strains to control spotted wing drosophila populations. J Appl Ecol 55(5):2408-2418

Clancy DJ, Hoffmann AA (1998) Environmental effects on cytoplasmic incompatibility and bacterial load in Wolbachia-infected Drosophila simulans. Entomol Exp Appl 86(1):13-24

Conner WR, Blaxter ML, Anfora G, Ometto L, Rota-Stabelli O, Turelli M (2017) Genome comparisons indicate recent transfer of wRi-like Wolbachia between sister species Drosophila suzukii and D. subpulchrella. Ecol Evol 7(22):9391-9404

Cooper BS, Ginsberg PS, Turelli M, Matute DR (2017) Wolbachia in the Drosophila yakuba complex: pervasive frequency variation and weak cytoplasmic incompatibility, but no apparent effect on reproductive isolation. Genetics 205(1):333-351

Crawley MJ (2007) The R book. John Wiley \& Sons, Ltd, Chichester, England

Dobson SL, Marsland EJ, Rattanadechakul W (2002) Mutualistic Wolbachia infection in Aedes albopictus: accelerating cytoplasmic drive. Genetics 160(3):1087-1094

Duron O, Bouchon D, Boutin S, Bellamy L, Zhou LQ, Engelstadter J et al. (2008) The diversity of reproductive parasites among arthropods: Wolbachia do not walk alone. BMC Biol 6:27

Engelstadter J, Hurst GDD (2009) The ecology and evolution of microbes that manipulate host reproduction. Annu Rev Ecol Evol Syst 40:127-149 
Engelstadter J, Telschow A (2009) Cytoplasmic incompatibility and host population structure. Heredity 103(3):196-207

Enigl M, Schausberger P (2007) Incidence of the endosymbionts Wolbachia, Cardinium and Spiroplasma in phytoseiid mites and associated prey. Exp Appl Acarol 42(2):75-85

Ferguson LV, Dhakal P, Lebenzon JE, Heinrichs DE, Bucking C, Sinclair BJ (2018) Seasonal shifts in the insect gut microbiome are concurrent with changes in cold tolerance and immunity. Funct Ecol 32(10):2357-2368

Fragata I, Lopes-Cunha M, Barbaro M, Kellen B, Lima M, Faria GS et al. (2016) Keeping your options open: maintenance of thermal plasticity during adaptation to a stable environment. Evolution 70 (1):195-206

Fragata I, Simoes P, Lopes-Cunha M, Lima M, Kellen B, Barbaro M et al. (2014) Laboratory selection quickly erases historical differentiation. PLoS ONE 9(5):e96227

Frago E, Dicke M, Godfray HCJ (2012) Insect symbionts as hidden players in insect-plant interactions. Trends Ecol Evol 27 (12):705-711

Francuski L, Djurakic M, Ludoski J, Hurtado P, Perez-Banon C, Stahls $G$ et al. (2014) Shift in phenotypic variation coupled with rapid loss of genetic diversity in captive populations of Eristalis tenax (Diptera: Syrphidae): consequences for rearing and potential commercial use. J Econ Entomol 107(2):821-832

Gibson CM, Hunter MS (2010) Extraordinarily widespread and fantastically complex: comparative biology of endosymbiotic bacterial and fungal mutualists of insects. Ecol Lett 13(2):223-234

Gotoh T, Noda H, Hong XY (2003) Wolbachia distribution and cytoplasmic incompatibility based on a survey of 42 spider mite species (Acari: Tetranychidae) in Japan. Heredity 91(3):208-216

Gotoh T, Noda H, Ito S (2007a) Cardinium symbionts cause cytoplasmic incompatibility in spider mites. Heredity 98(1):13-20

Gotoh T, Sugasawa J, Noda H, Kitashima Y (2007b) Wolbachiainduced cytoplasmic incompatibility in Japanese populations of Tetranychus urticae (Acari: Tetranychidae). Exp Appl Acarol 42 (1):1-16

Hamilton WD (1967) Extraordinary sex ratios. Science 156 (3774):477-488

Hamm CA, Begun DJ, Vo A, Smith CC, Saelao P, Shaver AO et al. (2014) Wolbachia do not live by reproductive manipulation alone: infection polymorphism in Drosophila suzukii and $D$. subpulchrella. Mol Ecol 23(19):4871-4885

Hancock PA, Godfray HCJ (2012) Modelling the spread of Wolbachia in spatially heterogeneous environments. J R Soc Interface 9 (76):3045-3054

Hoffmann AA, Hallas R, Sinclair C, Partridge L (2001) Rapid loss of stress resistance in Drosophila melanogaster under adaptation to laboratory culture. Evolution 55(2):436-438

Hoffmann AA, Iturbe-Ormaetxe I, Callahan AG, Phillips B, Billington $\mathrm{K}$, Axford JK et al. (2014) Stability of the wMel Wolbachia Infection following invasion into Aedes aegypti populations. PLoS Negl Trop Dis 8(9):e3115

Hoffmann AA, Ross PA (2018) Rates and patterns of laboratory adaptation in (mostly) insects. J Econ Entomol 111(2):501-509

Hoffmann AA, Turelli M, Harshman LG (1990) Factors affecting the distribution of cytoplasmic incompatibility in Drosophila simulans. Genetics 126(4):933-948

Hopkins SR, Wojdak JM, Belden LK (2017) Defensive symbionts mediate host-parasite interactions at multiple scales. Trends Parasitol 33(1):53-64

Hurst LD, Atlan A, Bengtsson BO (1996) Genetic conflicts. Q Rev Biol 71(3):317-364

Ishmael N, Hotopp JCD, Ioannidis P, Biber S, Sakamoto J, Siozios S et al. (2009) Extensive genomic diversity of closely related Wolbachia strains. Microbiol-Sgm 155:2211-2222
Jansen VAA, Turelli M, Godfray HCJ (2008) Stochastic spread of Wolbachia. Proc R Soc B 275(1652):2769-2776

Kaur R, Siozios S, Miller WJ, Rota-Stabelli O (2017) Insertion sequence polymorphism and genomic rearrangements uncover hidden Wolbachia diversity in Drosophila suzukii and D. subpulchrella. Sci Rep 7(1):14815

Keller GP, Windsor DM, Saucedo JM, Werren JH (2004) Reproductive effects and geographical distributions of two Wolbachia strains infecting the Neotropical beetle, Chelymorpha alternans Boh. (Chrysomelidae, Cassidinae). Mol Ecol 13(8):2405-2420

Kriesner P, Hoffmann AA, Lee SF, Turelli M, Weeks AR (2013) Rapid sequential spread of two Wolbachia variants in Drosophila simulans. PLoS Pathog 9(9):e1003607

Leftwich PT, Bolton M, Chapman T (2016) Evolutionary biology and genetic techniques for insect control. Evolut Appl 9(1):212-230

LePage DP, Metcalf JA, Bordenstein SR, On JM, Perlmutter JI, Shropshire JD et al. (2017) Prophage WO genes recapitulate and enhance Wolbachia-induced cytoplasmic incompatibility. Nature 543(7644):243-247

Lindsey ARI, Rice DW, Bordenstein SR, Brooks AW, Bordenstein SR, Newton ILG (2018) Evolutionary genetics of cytoplasmic incompatibility genes cifA and cifB in prophage WO of Wolbachia. Genome Biol Evolution 10(2):434-451

Liu Y, Miao H, Hong XY (2006) Distribution of the endosymbiotic bacterium Cardinium in Chinese populations of the carmine spider mite Tetranychus cinnabarinus (Acari: Tetranychidae). J Appl Entomol 130(9-10):523-529

Macke E, Magalhães S, Bach F, Olivieri I (2011) Experimental evolution of reduced sex ratio adjustment under local mate competition. Science 334(6059):1127-1129

Matos M, Simões P, Santos MA, Seabra SG, Faria GS, Vala F et al. (2015) History, chance and selection during phenotypic and genomic experimental evolution: replaying the tape of life at different levels. Front Genet 6:71

Mercot H, Charlat S (2004) Wolbachia infections in Drosophila melanogaster and D. simulans: polymorphism and levels of cytoplasmic incompatibility. Genetica 120(1-3):51-59

Moran NA, McCutcheon JP, Nakabachi A (2008) Genomics and evolution of heritable bacterial symbionts. Annu Rev Genet 42:165-190

Narita S, Nomura M, Kageyama D (2007) Naturally occurring single and double infection with Wolbachia strains in the butterfly Eurema hecabe: transmission efficiencies and population density dynamics of each Wolbachia strain. FEMS Microbiol Ecol 61 (2):235-245

Nguyen TH, Le Nguyen H, Nguyen TY, Vu SN, Tran ND, Le TN et al. (2015) Field evaluation of the establishment potential of wMelPop Wolbachia in Australia and Vietnam for dengue control. Parasite Vector 8:563

Oliver KM, Smith AH, Russell JA (2014) Defensive symbiosis in the real world -advancing ecological studies of heritable, protective bacteria in aphids and beyond. Funct Ecol 28(2):341-355

Perlman SJ, Kelly SE, Hunter MS (2008) Population biology of cytoplasmic incompatibility: maintenance and spread of Cardinium symbionts in a parasitic wasp. Genetics 178(2):1003-1011

Perrot-Minnot MJ, Cheval B, Migeon A, Navajas M (2002) Contrasting effects of Wolbachia on cytoplasmic incompatibility and fecundity in the haplodiploid mite Tetranychus urticae. J Evol Biol 15(5):808-817

Poinsot D, Bourtzis K, Markakis G, Savakis C, Mercot H (1998) Wolbachia transfer from Drosophila melanogaster into $D$. simulans: Host effect and cytoplasmic incompatibility relationships. Genetics 150(1):227-237

Rasgon JL, Scott TW (2003) Wolbachia and cytoplasmic incompatibility in the california Culex pipiens mosquito species complex: 
Parameter estimates and infection dynamics in natural populations. Genetics 165(4):2029-2038

Raychoudhury R, Baldo L, Oliveira D, Werren JH (2009) Modes of acquisition of Wolbachia: horizontal transfer, hybrid introgression, and codivergence in the Nasonia species complex. Evolution 63(1):165-183

Reuter M, Lehmann L, Guillaume F (2008) The spread of incompatibility-inducing parasites in sub-divided host populations. BMC Evol Biol 8:134

Reynolds KT, Hoffmann AA (2002) Male age, host effects and the weak expression or nonexpression of cytoplasmic incompatibility in Drosophila strains infected by maternally transmitted Wolbachia. Genetical Res 80(2):79-87

Ros VID, Breeuwer JAJ (2009) The effects of, and interactions between, Cardinium and Wolbachia in the doubly infected spider mite Bryobia sarothamni. Heredity 102(4):413-422

Ros VID, Fleming VM, Feil EJ, Breeuwer JAJ (2012) Diversity and recombination in Wolbachia and Cardinium from Bryobia spider mites. BMC Microbiol 12(Suppl 1):S13

Ross PA, Axford JK, Richardson KM, Endersby-Harshman NM, Hoffmann AA (2017a) Maintaining Aedes aegypti mosquitoes infected with Wolbachia. J Vis Exp (126):e56124. https://doi.org/ $10.3791 / 56124$

Ross PA, Wiwatanaratanabutr I, Axford JK, White VL, EndersbyHarshman NM, Hoffmann AA (2017b) Wolbachia infections in Aedes aegypti differ markedly in their response to cyclical heat stress. PLoS Pathog 13(1):17

Schmidt TL, Barton NH, Rasic G, Turley AP, Montgomery BL, Iturbe-Ormaetxe I et al. (2017) Local introduction and heterogeneous spatial spread of dengue-suppressing Wolbachia through an urban population of Aedes aegypti. PLoS Biol 15(5):e2001894

Sousa V, Zélé F, Rodrigues LR, Godinho DP, Charlery M, Magalhães S (2019) Rapid host-plant adaptation in the herbivorous spider mite Tetranychus urticae occurs at low cost. Curr Opin Insect Sci 36:82-89

Staudacher H, Schimmel BCJ, Lamers MM, Wybouw N, Groot AT, Kant MR (2017) Independent effects of a herbivore's bacterial symbionts on its performance and induced plant defences. Int $\mathrm{J}$ Mol Sci 18(1):182

Suh E, Sim C, Park J-J, Cho K (2015) Inter-population variation for Wolbachia induced reproductive incompatibility in the haplodiploid mite Tetranychus urticae. Exp Appl Acarol 65(1):55-71

Sumi T, Miura K, Miyatake T (2017) Wolbachia density changes seasonally amongst populations of the pale grass blue butterfly, Zizeeria maha (Lepidoptera: Lycaenidae). PLoS ONE 12(4):10

Sun JX, Guo Y, Zhang X, Zhu WC, Chen YT, Hong XY (2016) Effects of host interaction with Wolbachia on cytoplasmic incompatibility in the two-spotted spider mite Tetranychus urticae. Biol J Linn Soc 119(1):145-157

Tamura K, Peterson D, Peterson N, Stecher G, Nei M, Kumar S (2011) MEGA5: molecular evolutionary genetics analysis using maximum likelihood, evolutionary distance, and maximum parsimony methods. Mol Biol Evol 28(10):2731-2739

Toju H, Fukatsu T (2011) Diversity and infection prevalence of endosymbionts in natural populations of the chestnut weevil: relevance of local climate and host plants. Mol Ecol 20 (4):853-868

Turelli M, Hoffmann AA (1995) Cytoplasmic incompatibility in Drosophila simulans-dynamics and parameter estimates from natural-populations. Genetics 140(4):1319-1338

Vala F, Van Opijnen T, Breeuwer JAJ, Sabelis MW (2003) Genetic conflicts over sex ratio: mite-endosymbiont interactions. Am Nat 161(2):254-266

Vala F, Weeks A, Claessen D, Breeuwer JAJ, Sabelis MW (2002) Within- and between-population variation for Wolbachia-induced reproductive incompatibility in a haplodiploid mite. Evolution 56 (7):1331-1339

Van Opijnen T, Breeuwer JAJ (1999) High temperatures eliminate Wolbachia, a cytoplasmic incompatibility inducing endosymbiont, from the two-spotted spider mite. Exp Appl Acarol 23 (11):871-881

Vavre F, Fleury F, Lepetit D, Fouillet P, Bouletreau M (1999) Phylogenetic evidence for horizontal transmission of Wolbachia in host-parasitoid associations. Mol Biol Evol 16(12):1711-1723

Vavre F, Fleury F, Varaldi J, Fouillet P, Bouletreau M (2000) Evidence for female mortality in Wolbachia-mediated cytoplasmic incompatibility in haplodiploid insects: epidemiologic and evolutionary consequences. Evolution 54(1):191-200

Vavre F, Fleury F, Varaldi J, Fouillet P, Bouletreau M (2002) Infection polymorphism and cytoplasmic incompatibility in HymenopteraWolbachia associations. Heredity 88:361-365

Weeks AR, Reynolds KT, Hoffmann AA, Mann H (2002) Wolbachia dynamics and host effects: what has (and has not) been demonstrated? Trends Ecol Evol 17(6):257-262

Weinert LA, Araujo-Jnr EV, Ahmed MZ, Welch JJ (2015) The incidence of bacterial endosymbionts in terrestrial arthropods. Proc R Soc Lond 282(1807):20150249

Werren JH, Beukeboom LW (1998) Sex determination, sex ratios, and genetic conflict. Annu Rev Ecol Syst 29:233-261

Xie RR, Chen XL, Hong XY (2011) Variable fitness and reproductive effects of Wolbachia infection in populations of the two-spotted spider mite Tetranychus urticae Koch in China. Appl Entomol Zool 46(1):95-102

Xie RR, Zhou LL, Zhao ZJ, Hong XY (2010) Male age influences the strength of Cardinium-induced cytoplasmic incompatibility expression in the carmine spider mite Tetranychus cinnabarinus. Appl Entomol Zool 45(3):417-423

Yu MZ, Zhang KJ, Xue XF, Hong XY (2011) Effects of Wolbachia on mtDNA variation and evolution in natural populations of Tetranychus urticae Koch. Insect Mol Biol 20(3):311-321

Zeh JA, Bonilla MM, Adrian AJ, Mesfin S, Zeh DW (2012) From father to son: transgenerational effect of tetracycline on sperm viability. Sci Rep 2:375

Zélé F, Santos I, Olivieri I, Weill M, Duron O, Magalhães S (2018a) Endosymbiont diversity and prevalence in herbivorous spider mite populations in South-Western Europe. FEMS Microbiol Ecol 94(4):fiy015

Zélé F, Santos JL, Godinho DP, Magalhães S (2018b) Wolbachia both aids and hampers the performance of spider mites on different host plants. FEMS Microbiol Ecol 94(12):fiy 187

Zélé F, Weill M, Magalhães S (2018c) Identification of spider-mite species and their endosymbionts using multiplex PCR. Exp Appl Acarol 74:123-138

Zhang YK, Chen YT, Yang K, Qiao GX, Hong XY (2016) Screening of spider mites (Acari: Tetranychidae) for reproductive endosymbionts reveals links between co-infection and evolutionary history. Sci Rep 6:27900

Zhang YK, Ding XL, Zhang KJ, Hong XY (2013a) Wolbachia play an important role in affecting mtDNA variation of Tetranychus truncatus (Trombidiformes: Tetranychidae). Environ Entomol 42 (6): 1240-1245

Zhang YK, Zhang KJ, Sun JT, Yang XM, Ge C, Hong XY (2013b) Diversity of Wolbachia in natural populations of spider mites (genus Tetranychus): Evidence for complex infection history and disequilibrium distribution. Microb Ecol 65(3):731-739

Zhao DX, Chen DS, Ge C, Gotoh T, Hong XY (2013a) Multiple infections with Cardinium and two strains of Wolbachia in the spider mite Tetranychus phaselus Ehara: revealing new forces driving the spread of Wolbachia. PLoS ONE 8(1):e54964 
Zhao DX, Zhang XF, Hong XY (2013b) Host-symbiont interactions in spider mite Tetranychus truncates doubly infected with Wolbachia and Cardinium. Environ Entomol 42 (3):445-452

Zhu LY, Zhang KJ, Zhang YK, Ge C, Gotoh T, Hong XY (2012) Wolbachia strengthens Cardinium-induced cytoplasmic incompatibility in the spider mite Tetranychus piercei McGregor. Curr Microbiol 65(5):516-523

Zhu Y-X, Song Y-L, Zhang Y-K, Hoffmann AA, Zhou J-C, Sun J-T et al. (2018) Incidence of facultative bacterial endosymbionts in spider mites associated with local environment and host plant. Appl Environ Microbiol 84(6):e02546-02517 